\title{
Le metteur en scène et les autres ou de la difficulté du partage de l'auctorialité dans le théâtre public français
}

The producer and the others, or the difficulty of collaborative editorialization in the French public theatre

Bérénice Hamidi-Kim

\section{(2) OpenEdition}

Édition électronique

URL : http://journals.openedition.org/tangence/1053

ISSN : 1710-0305

Éditeur :

Université du Québec à Rimouski, Université du Québec à Trois-Rivières

Édition imprimée

Date de publication : 1 décembre 2019

Pagination : 61-79

ISSN : 1189-4563

Référence électronique

Bérénice Hamidi-Kim, «Le metteur en scène et les autres ou de la difficulté du partage de l'auctorialité dans le théâtre public français », Tangence [En ligne], 121 | 2019, mis en ligne le 01 décembre 2020, consulté le 22 décembre 2020. URL : http://journals.openedition.org/tangence/1053

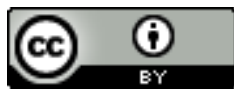

La revue Tangence est mise à disposition selon les termes de la Licence Creative Commons Attribution 4.0 International. 
Tangence $\square \mathrm{n}^{\circ}$ 121, 2019, p. 61-79.

\section{Le metteur en scène et les autres ou de la difficulté du partage de l'auctorialité dans le théâtre public français}

Bérénice Hamidi-Kim

Université Lyon 2/Institut Universitaire de France

Le présent article vise à élucider les causes et à examiner les effets d'un paradoxe caractéristique du monde du spectacle vivant subventionné en France: alors que les œuvres scéniques ont pour particularité, par rapport à d'autres mondes de l'art, de reposer sur le travail collectif d'une grande diversité de corps de métiers, artistiques et techniques, la reconnaissance économique, sociale et symbolique est pour l'essentiel captée par la figure du metteur en scène. Pourquoi une telle concentration des pouvoirs entre les mains de ce type d'artiste? Par quoi passe-t-elle? Quelles sont les stratégies aujourd'hui mises en œuvre au sein des collectifs de travail artistique que sont les compagnies pour atténuer cette concentration? Comment sont-elles perçues par ceux qui financent la production et la diffusion des spectacles? De fait, dans le cadre d'une économie subventionnée comme celle du «théâtre public», et d'un secteur socio-économique dans lequel les compagnies sont soumises aux attentes des producteurs et des diffuseurs que sont les théâtres, selon les lois de l'offre et de la demande, l'analyse du fonctionnement interne de ces compagnies passe par une prise en compte de ces demandes extérieures, implicites ou explicites. Cette réflexion prendra appui sur plusieurs enquêtes de terrain: une série d'entretiens avec des directeurs de compagnie menés entre 2015 et 2017, en partie pour la rubrique "chaîne de productions» de la revue thaêtre, en partie dans le cadre d'un programme de recherche sur 
les «"nouvelles" organisations du travail en compagnie» coordonné avec Séverine Ruset ${ }^{1}$. Elle s'appuie également sur l'observation des commissions d'aide au projet des $\mathrm{DRAC}^{2}$ et sur l'étude du processus de création de quelques compagnies de théâtre et de cirque.

On peut considérer que le processus de captation du pouvoir passe pour l'essentiel par la transfiguration de la figure du metteur en scène en auteur de l'ouvre scénique ${ }^{3}$. Il s'agit là d'un phénomène ancien, datant au moins des années 1880 et désormais bien renseigné par les historiens du théâtre, qui est étroitement lié à l'«invention » de la mise en scène moderne ${ }^{4}$. Mais, s'il prend sa source dans cette transformation à la fois esthétique et symbolique, ce phénomène, pourvu de conséquences matérielles, socio-économiques notamment, s'est trouvé renforcé par la progressive structuration institutionnelle du théâtre sous les espèces du «théâtre populaire» puis du «théâtre public», entre la fin du XIx $x^{e}$ et les années $1960^{5}$. Sans faire ici une histoire au long cours des transformations de la figure institutionnelle du metteur en scène du théâtre public, il faut rappeler le tournant des années 1970. Alors que, jusque-là, les metteurs en scène-chefs de troupes ne se définissaient jamais simplement ni essentiellement comme artistes, auteurs d'une œuvre ${ }^{6}$, mais toujours aussi et surtout comme "animateurs», pour reprendre l'expression popularisée par l'illustre Jean Vilar, la crise du modèle de la décentralisation théâtrale

1. Voir Bérénice Hamidi-Kim et Séverine Ruset (dir.), Troupes, compagnies, collectifs dans les arts vivants: organisation du travail, processus de création et conjonctures, Paris, L'Entretemps, 2018.

2. Il s'agit des Directions Régionales des Affaires Culturelles qui subventionnent la culture au niveau des régions.

3. Voir notamment Marco Consolini et coll., «Le metteur en scène comme auteur du théâtre. Zola, Gourmont, Claudel, Pirandello, Ibsen, Duras, Beckett, Handke, Gabily...", Études théâtrales, $\mathrm{n}^{\text {os }} 38-39$ (La réinvention du drame (sous l'influence de la scène), dir. Jean-Pierre Sarrazac et Catherine Naugrette), 2007, p. 31-41 et Bénédicte Boisson, Alice Folco et Ariane Martinez, Histoire de la mise en scène, Paris, Presses universitaires de France, 2010.

4. Voir Études théâtrales, $\mathrm{n}^{\mathrm{os}}$ 15-16 (Mise en crise de la forme dramatique 1880-1910, dir. Jean-Pierre Sarrazac), 1999.

5. Voir Bérénice Hamidi-Kim, «Théâtres populaires (républicain+socialiste+paternaliste) $=$ théâtre public? De la diversité historique des grammaires du théâtre populaire en France, ou comment les études théâtrales écrivent l'histoire du théâtre», dans Olivier Bara (dir.), Théâtre et peuple de Louis-Sébastien Mercier à Firmin Gémier, Paris, Classiques Garnier, coll. «Rencontres», série "Études théâtrales", 2017, p. 529-546.

6. Voir à ce sujet Robert Abirached (dir.), La décentralisation théâtrale, vol. 1, Le premier âge: 1945-1958, Arles, ANRAT/Actes Sud Papiers, 2005. 
qui s'est ouverte en Mai 68 a consacré le "pouvoir aux créateurs» à la fois contre les pouvoirs publics locaux et contre leur ancienne mission de chef de troupe - ou de collectif — et d'évangélisateur esthétique et démocratique des foules de citoyens-spectateurs.

Cette contextualisation historique étant rapidement posée, je partirai de considérations formulées par Foucault dans le texte de 1969 "Qu'est-ce qu'un auteur?" », dans lequel il distingue différentes caractéristiques de la «fonction-auteur». La première est qu'elle s'arrime irréductiblement à la production d'une ouvre: c'est en tant qu'elle est productrice d'un bien matériel, l'œuvre, que la figure de l'auteur renvoie à un système institutionnel et juridique et s'inscrit dans un circuit de propriété - qui dit auteur dit droits d'auteur. Ensuite, Foucault insiste sur l'instabilité historique de la fonction d'autorité du nom de l'auteur: référer une œuvre à un nom qui la signe n'est pas une démarche qui a toujours existé: longtemps les œuvres ont pu être anonymes. Enfin, Foucault caractérise l'auteur comme la figure émanant de l'œuvre, de la cohérence stylistique et conceptuelle des différents textes écrits par une même personne. L'auteur, en ce sens, se crée toujours dans l'après-coup: il s'agit d'une figure constituée par le regard et par la plume d'autres auteurs (critiques, universitaires) ${ }^{8}$. Dans le théâtre subventionné français, le metteur en scène peut, à tous ces titres, être considéré comme un auteur et c'est en cela qu'il concentre les pouvoirs économique, social et symbolique. Ainsi, même si l'acte de création est toujours une collaboration entre différents corps de métiers artistiques (auteur de texte parfois différent, interprètes, et collaborateurs artistiques - vidéo, son, lumière, etc.) et techniques, le plus souvent, le metteur en scène se considère et est considéré comme l'auteur des spectacles créés, à la fois au sens où il détient l'autorité artistique dans le temps du processus de création des spectacles et au sens où il incarne la compagnie et sa ligne artistique aux yeux de l'extérieur.

7. Michel Foucault, "Qu'est-ce qu'un auteur?», dans Dits et écrits I, 1954-1975, Paris, Gallimard, 2001, p. 789-821.

8. Il s'agit donc d'un problème d'identité sociale. On pourrait transposer à la scène théâtrale les analyses de José-Luis Diaz, qui distingue trois modes d'existence de l'auteur sur la scène littéraire: l'auteur réel, pris dans son identité sociale et physique d'auteur, l'auteur textuel — à la fois auteur du livre et sujet textuel, sujet du discours - et l'auteur imaginaire: l'auteur tel qu'il se représente/est représenté (L'écrivain imaginaire. Scénographies auctoriales à l'époque romantique, Paris, Honoré Champion, coll. «Romantisme et modernités», 2007). 
Il est donc l'auteur au sens où il signe l'acte de création. Comme le formule David Lescot, lui-même auteur et metteur en scène, «l'écriture n'est pas que la matière textuelle, c'est aussi la construction» du spectacle, ce qui explique que "quand le metteur en scène fait un travail de construction, il fait un travail d'auteur ${ }^{9} »$. La formule «écrivain de plateau ${ }^{10}$ » forgée par Bruno Tackels ou l'élargissement fortement promu par la Société des auteurs et compositeurs dramatiques (SACD) du droit d'auteur aux ouvres scéniques attestent du consensus existant aujourd'hui ${ }^{11}$, à la fois dans le champ universitaire et dans le champ professionnel du spectacle vivant, pour faire de la figure du metteur en scène l'auteur des spectacles et l'instance primordiale d'auctorialité et donc, de captation de la reconnaissance que conferre ce statut - dans le même temps que la légitimation du rôle de metteur en scène est ce qui a permis à la mise en scène de théâtre d'être élevée au rang d'art ${ }^{12}$.

Cette prééminence a une histoire, nous l'avons vu, mais elle tient aussi à l'organisation du travail artistique aujourd'hui en France. À la différence d'autres pays (telle l'Angleterre, dont le système de production est davantage centré sur la figure de l'auteur de texte dramatique) ou d'autres mondes de l'art (tel le cinéma hollywoodien où les acteurs bankable détiennent un pouvoir considérable et supérieur aux réalisateurs, puisque c'est sous leur nom et non celui du réalisateur qu'un projet peut être produit), le pouvoir économique et symbolique est en effet détenu par les metteurs en scène, directeurs artistiques des "compagnies», ces instances qui formulent les demandes de subvention auprès des pouvoirs publics, qu'il s'agisse de demandes d' «aide au projet» (pour la production d'un spectacle) ou de «conventionnement» (demande de subvention pluriannuelle, accessible aux compagnies qui ont déjà à leur actif

9. David Lescot, entretien personnel avec Bérénice Hamidi-Kim, Paris, 23 janvier 2015.

10. Bruno Tackels, Les écritures de plateau, Besançon, Les Solitaires intempestifs, 2015.

11. La tendance est cependant bien plus ancienne et date elle aussi du tournant du XIX ${ }^{e}$ siècle. Voir notamment Marco Consolini et coll., «Le metteur en scène comme auteur du théâtre. Zola, Gourmont, Claudel, Pirandello, Ibsen, Duras, Beckett, Handke, Gabily... », art. cité, p. 31-41.

12. Voir Serge Proust, «Les metteurs en scène de théâtre entre réussite sociale et remise en cause ontologique», dans Nathalie Heinich, Roberta Shapiro (dir.), De l'artification. Enquêtes sur le passage à l'art, Paris, EHESS, coll. «Cas de figure», 2012, p. 95-111. 
quelques spectacles reconnus produits et ayant tourné dans le cadre des institutions du théâtre public). De même, à quelques exceptions près, la direction des théâtres subventionnés les plus prestigieux et les mieux dotés que sont les Centres Dramatiques Nationaux (CDN) et les Théâtres Nationaux est réservée aux metteurs en scène, et non aux autres artistes (auteurs, acteurs).

J'ai fait le choix de centrer mon attention sur ce qui se joue au sein des compagnies indépendantes et non des structures de production-diffusion que sont les théâtres subventionnés, pour plusieurs raisons. D'abord et avant tout, parce qu'il s'agit du cadre majoritaire dans et par lequel les spectacles sont produits et diffusés — il y a de fait plus de 600 compagnies professionnelles alors qu'il n'existe qu'une quarantaine de CDN et cinq Théâtres Nationaux. Ensuite, parce que les compagnies indépendantes constituent un site d'observation privilégié de la crise non seulement économique, mais aussi idéologique du spectacle vivant. Ce sont elles qui sont accusées, par certains financeurs et commentateurs du spectacle vivant subventionné, d'être responsables des maux économiques du secteur, dont elles sont pourtant les principales à souffrir, au motif que leur prolifération serait cause du déséquilibre du modèle économique du secteur - selon un argument d'autant plus surprenant que les compagnies bénéficient de subventions mais ne les attribuent pas. Mais les compagnies concentrent aussi l'espoir, formulé par les artistes et encouragé par les pouvoirs publics ${ }^{13}$, d'un renouveau du socle idéologique du théâtre public. C'est en leur sein qu'est formulé l'appel à faire "autrement», synonyme pour les artistes d'une volonté de déjouer, ou du moins d'atténuer un rapport de force structurel entre ceux qui financent (subventionneurs et producteurs-programmateurs) et ceux qui sont financés... et synonyme pour les financeurs d'un renouveau bienvenu du socle de légitimité du théâtre public.

De fait, si crise du secteur il y a, il s'agit d'une crise économique qui redouble les effets d'une crise de légitimité trouvant son origine en Mai $1968^{14}$. Le théâtre ne s'est jamais remis de la prise de

13. Voir Bérénice Hamidi-Kim et Séverine Ruset, «Introduction», dans Troupes, compagnies, collectifs, ouvr. cité.

14. Voir Bérénice Hamidi-Kim, Les cités du théâtre politique en France depuis 1989, préface de Luc Boltanski, Montpellier, L'Entretemps, coll. "Champ théâtral», 2013. 
conscience du fait que la mission de «démocratisation des publics» échouait partiellement, non par manque de volontarisme des théâtres (centres dramatiques et maisons de la culture) pour la conquête de nouveaux spectateurs, mais parce que l'ambition historique fondatrice des politiques culturelles françaises - articuler élargissement social des publics et exigence esthétique - n'allait pas de soi et ne pouvait faire du théâtre une pratique culturelle majoritaire et de masse. On pourrait presque dire que le secteur ne s'est jamais remis de cette mise en doute, malgré l'embellie des années 1980, et que c'est pour cette raison que les registres de justification du financement public du théâtre se sont diversifiés depuis ${ }^{15}$. La stratégie des professionnels du secteur comme des pouvoirs publics, inconsciente certes mais agissante, a consisté à déplacer le regard de la salle vers la scène et les coulisses, à ne plus focaliser le discours sur le caractère émancipateur du théâtre uniquement autour des effets potentiels sur les spectateurs, et à mettre en valeur celui de l'acte de création. C'est ce qui explique, d'une part, la faveur des projets dits "participatifs» et la valorisation de l'éducation artistique et, d'autre part, la mise en avant du caractère émancipateur du travail artistique dans ce secteur socio-professionnel. De fait, les professions artistiques correspondent à un travail de type vocationnel dans lequel l'individu met de lui-même et s'épanouit, et portent, pour ceux qui les exercent comme pour le reste de la société, le rêve d'une possible actualisation $\mathrm{du}$ «travail libre» théorisé par Marx. Mais c'est aussi l'organisation concrète du travail artistique dans le champ du spectacle vivant qui est prise en considération dans ce discours qui valorise le régime de l'intermittence ${ }^{16}$ et le travail en compagnie, en tant que ce régime et ce type d'organisation semblent cristalliser, respectivement à l'échelle individuelle et à l'échelle collective, le rêve d'une organisation du travail libérée du lien de subordination caractéristique du travail salarié classique. Il importe de préciser que ce renouveau du socle de légitimation du théâtre par sa valeur démocratique, civique, émancipatrice non plus seulement pour les publics (réels ou potentiels),

15. Voir notamment Bérénice Hamidi-Kim, «Développement durable (culturel) et démocratie culturelle: nouveaux/derniers avatars de la grandiloquence du théâtre public?», Registres, $n^{\circ} 18$ (Théâtre et développement durable, dir. Daniel Urrutiaguer), 2015, p. 34-43.

16. Voir notamment, pour exemple de ce discours, Antonella Corsani et Maurizio Lazzarato, Intermittents et précaires, Paris, Éditions Amsterdam, 2008. 
mais aussi pour ceux qui le font, a lui aussi été fortement déstabilisé depuis une dizaine d'années. De fait, ont surgi avec force autant qu'avec raison des mises en cause d'inégalités structurelles à l'œuvre dans le champ de la production artistique. Ces remises en cause se sont fondées sur des études statistiques réalisées par le ministère de la Culture même ${ }^{17}$, puis par des organismes professionnels ${ }^{18}$ et des associations - l'association $\mathrm{H} / \mathrm{F}^{19}$ et plus récemment l'association Décoloniser les arts ${ }^{20}$. Curieusement, les inégalités économiques et symboliques structurelles entre corps de métier n'ont pour leur part jamais fait l'objet d'une thématisation dans le débat public. Tout au plus arrive-t-il exceptionnellement, quand un metteur en scène occupe la place de directeur de théâtre, qu'un conflit social aboutisse à dénoncer ce qui apparaît à certains comme un abus de pouvoir de la part du directeur - la grève d'une partie du personnel du Théâtre de la Commune d'Aubervilliers en désaccord avec la politique managériale de la directrice du théâtre Marie-José Malis en est un exemple tout récent. Mais c'est alors en tant que «patron d'entreprise» que le directeur de théâtre est mis en cause. Curieusement, les directeurs de compagnies ne sont jamais considérés comme tels, et l'hégémonie de leur pouvoir ne semble pas patente. Il me semble important d'insister sur cette inégalité structurelle et de considérer qu'elle peut être jugée injuste. De fait, l'enquête de terrain menée sur les dispositifs de sélection au sein des commissions DRAC ${ }^{21}$ a révélé l'importance

17. Voir en particulier Reine Prat, «Pour l'égal accès des hommes et des femmes aux postes de responsabilité, aux lieux de décision, à la maitrise de la représentation", Mission ÉgalitéS [En ligne], mis en ligne le $1^{\text {er }}$ mai 2006, consulté le 31 juillet 2019, et «De l'interdit à l'empêchement», Mission ÉgalitéS [En ligne], mis en ligne en mai 2009, consulté le 31 juillet 2019, URL: http://www.culture.gouv. fr/Espace-documentation/Rapports/Mission-EgaliteS.

18. Voir la brochure "Où sont les femmes?", éditée depuis 2012 par la SACD (Société des auteurs et compositeurs dramatiques).

19. L'association H/F, pour l'égalité Hommes/Femmes dans le spectacle vivant, est née en Rhône-Alpes en 2008, à la suite du rapport de Reine Prat en 2006, puis des collectifs se sont formés dans différentes régions jusqu’à ce que se constitue, à l'été 2011, une Fédération interrégionale du Mouvement H/F.

20. L'association Décoloniser les arts, créée en décembre 2015, réunit des artistes "concernés» autant qu'alliés de cette cause, qui ont pour projet commun de dénoncer le manque de « diversité» et les discriminations à l'œuvre dans le spectacle vivant et d'œuvrer à transformer cet état de fait.

21. Cette enquête a été entamée par une série d'entretiens réalisés avec Daniel Urrutiaguer dans le cadre du séminaire «Incertitude sur la valeur», co-organisé avec Luc Boltanski, Bruno Cousin, Emmanuel Didier, Arnaud Esquerre et Jeanne Lazarus, EHESS, 2011-2012. Nous avons interrogé essentiellement des acteurs 
non négligeable, dans l'évaluation des projets des compagnies par les experts, de la composition de l'équipe artistique, notamment les interprètes, l'auteur et parfois même l'équipe administrative. Il reste donc à comprendre à quoi tient cette prééminence de la figure du metteur en scène d'une part et, d'autre part, l'absence de remise en cause de cette prééminence. Les facteurs d'explication sont pluriels, mais ils tiennent notamment à la structuration juridique de la plupart des compagnies.

Une compagnie ${ }^{22}$ peut être définie comme une organisation, c'est-à-dire une entité regroupant des personnes en vue de la réalisation coordonnée d'un objectif commun, en l'occurrence la création de spectacles, et qui se dote, pour atteindre cet objectif, de moyens humains, matériels, administratifs, juridiques, comptables, etc. ${ }^{23}$. En tant qu'organisations, ces entités sont marquées par des relations de pouvoir et de coopération qui se jouent à l'échelle d'un projet, mais aussi et surtout de l'ensemble des activités menées au long cours au sein d'une compagnie, entre, d'une part, le ou les artistes qui signent en définitive l'acte de création et, d'autre part, l'ensemble de l'équipe artistique, mais aussi technique et administrative. La plupart des compagnies ont le statut d'association loi de 1901 et, dans ce cadre juridique, le directeur artistique, presque toujours un metteur en scène, n'est pas officiellement leur employeur, puisqu'il est lui-même un employé de l'association — l'employeur au sens juridique, qui signe les contrats, est le président du bureau de l'association. Pour autant, c'est bien le metteur en scène le directeur artistique de la compagnie, et c'est bien lui qui prend les décisions

de la sélection nationaux, ministériels ou para-ministériels: une inspectrice du théâtre à la Direction générale de la création artistique (DGCA), un ancien conseiller du cabinet du ministère de la Culture, une conseillère de théatre à l'Office national de diffusion artistique (ONDA), des membres de différentes commissions DRAC — directeurs de DRAC, conseillers en théâtre, experts (non rémunérés par le Ministère mais sélectionnés par lui sur un principe de cooptation). Nous avons aussi rencontré un ancien membre de l'association Thécif (Théâtre et cinéma en Île-de-France, financée par la région Île-de-France, organisme promouvant la diffusion des spectacles sur le modèle de l'ONDA, mais à l'échelle régionale). J'ai ensuite poursuivi l'enquête sous la forme d'une observation des commissions d'aide au projet des DRAC Auvergne-Rhône-Alpes et Île-de-France et d'une nouvelle série d'entretiens à l'hiver 2015-2016.

22. Voir Bérénice Hamidi-Kim et Séverine Ruset (dir.), Troupes, compagnies, collectifs, ouvr. cité.

23. Selon la définition sociologique de l'organisation synthétisée par Lusin Bagla, Sociologie des organisations, Paris, La Découverte, coll. «Repères », 2003, p. 3-6. 
et qui choisit celles et ceux avec qui il va créer «son» spectacle. Ce décalage entre l'instance qui détient l'autorité juridique et celle qui détient l'autorité effective tend à masquer l'asymétrie radicale des positions entre le metteur en scène-directeur-artistique-porteur de projet et le reste des employés de la compagnie, et à renforcer le sentiment, chez toutes les parties impliquées, qu'il est question de se choisir et de se plaire pour créer ensemble de belles œuvres, et non d'être employé et d'employer dans le cadre d'un univers professionnel marqué par de fortes hiérarchies. Si l'écrasante majorité des compagnies $\left(96 \%{ }^{24}\right)$ est structurée en associations, quelques-unes font d'autres choix de structuration juridique, en optant pour une Société coopérative ouvrière de production (SCOP) ou, plus récemment, pour une Société coopérative d'intérêt collectif (SCIC). Ce choix d'une structuration juridique autre que celle d'une association s'explique toujours non seulement par le besoin de modifier le rapport de force extérieur avec les subventionneurs et les producteursprogrammateurs, mais aussi et surtout par le souci de reconfigurer et de décentrer le rapport de force interne à la compagnie. Il peut s'agir alors de réorganiser le partage de la décision à l'échelle de l'outil de production (partage des ressources financières, matérielles et réputationnelles de la compagnie) ou à l'échelle du processus de création. À quelque échelle qu'ils se situent, ces choix supposent de repenser le partage de l'autorité et de l'auctorialité.

Ces différences entre structures juridiques ne constituent, cela dit, qu'un des outils possibles, et pas toujours le plus adéquat, pour distinguer trois types principaux de compagnies. Le premier est centré sur la figure d'un directeur artistique, accompagné d'une équipe administrative très réduite mais stabilisée, et parfois d'une équipe technico-artistique (lumière, son, scénographie, vidéo, etc.), mobilisant selon ses besoins pour tel ou tel projet des équipes éphémères d'interprètes aux dimensions variables. On parle à son propos d' "adhocratie», terme qui désigne des organisations de travail souples et évolutives dont la taille et la forme s'ajustent en permanence selon les activités et les projets, et dont les membres sont polyvalents. Il serait plus juste en l'occurrence de parler d'adhocratie relative, dans la mesure où, dans la plupart des cas, ces compagnies

24. Voir Philippe Henry, «Les compagnies à direction artistique collective en France métropolitaine depuis 1980 », dans Raphaëlle Doyon et Guy Freixe (dir.), Les collectifs dans les arts vivants depuis 1980, Montpellier, L'Entretemps, 2014, p. 26. 
se composent aujourd'hui d'un noyau dur artistique, certes très resserré autour d'un (ou une) metteur·euse en scène, directeur-trice artistique de la compagnie, éventuellement accompagné.e d'un (ou plus souvent une) collaborateur-trice artistique proche, et parfois d'une personne ou d'une structure en charge de l'administration et de la production, quand la compagnie en a les moyens. Ce noyau dur s'entoure de trois autres types de partenaires: une équipe d'interprètes, plus ou moins fidélisée, le plus souvent reconfigurée selon les besoins de chaque projet; une équipe de collaborateurs artistiques et techniques (pour la scénographie, parfois la dramaturgie, la lumière, le son, l'image et la vidéo, etc.) avec lesquels l'engagement s'inscrit plus volontiers dans la durée, les fonctions d'administration, de gestion, de production et de diffusion étant occupées soit par une équipe de collaborateurs administratifs, directement rémunérés par la compagnie de façon plus ou moins ponctuelle, soit indirectement, par des bureaux de production. Cette forme standard de la compagnie est typique du système de production que les économistes nomment le combination system, qui a cours également dans le monde des entreprises culturelles. Je rappelle ici les thèses d'Alfred Berheim et Dominique Leroy ${ }^{25}$ pour qui la pénétration du monde du théâtre par le capitalisme dès le XIX $x^{\mathrm{e}}$ siècle s'est manifestée par le passage d'un système de troupes permanentes (le stock-system) à un système d'agencements flexibles (le combination system) dans lequel des équipes temporaires sont constituées sur mesure au profit d'une production théâtrale isolée. De fait, le type de compagnie majoritaire en France aujourd'hui relève de ce combination system et semble inspiré plus précisément par le "nouvel esprit du capitalisme», caractérisé selon Boltanski et Chiapello ${ }^{26}$ par une organisation du travail "par équipe et par projet», ainsi que par des techniques de management qui ont pour particularité de flouter le rapport hiérarchique. La prédominance de ce modèle invite à élargir aux compagnies le constat

25. Alfred L. Ernheim, The Business of Theatre. An Economic History of the American Theatre 1750-1932, New York, Benjamin Blom Inc., 1932 ; Dominique Leroy, Histoire des arts du spectacle en France: aspects économiques, politiques et esthétiques de la Renaissance à la Première Guerre mondiale, Paris, L'Harmattan, 1990 et Économie des arts du spectacle en France, Paris, L'Harmattan, 1992.

26. Luc Boltanski et Ėve Chiapello, Le nouvel esprit du capitalisme, Paris, Gallimard, 1999. 
formulé par Pierre-Michel Menger à propos de l'intermittence ${ }^{27}$, et à pointer ainsi l'ambivalence des arts vivants subventionnés, que l'on peut, dans une certaine mesure, analyser non seulement comme contre-modèle de l'organisation capitaliste actuelle du travail, mais aussi, à certains égards, comme pointe avancée de ce nouvel esprit du capitalisme.

Les deux autres types de compagnies peuvent se comprendre comme des tentatives de lutter contre le rythme effréné que suppose une production «par projet» et pour refuser la fausse horizontalité promise au sein de ce premier type de compagnie, dit «adhocratique». Un second type de compagnie revendique le modèle de la «troupe», caractéristique du stock system et d'une production inscrite dans le temps long d'une équipe artistique stabilisée, qui repose pour autant sur l'existence d'une asymétrie et d'une hiérarchie assumées entre le «chef de troupe » et le reste de l'équipe artistique. Seules quelques rares compagnies, telle la compagnie Louis Brouillard, dirigée par Joël Pommerat, ont non seulement le désir, mais les moyens financiers de concrétiser ce modèle qui suppose que les interprètes aient un employeur principal, voire exclusif. Les rares exemples de ce modèle, qui n'a plus forcément aujourd'hui les faveurs des artistes interprètes ni des pouvoirs publics, se trouvent donc surtout au sein des CDN et des Théâtres Nationaux (la troupe du TNP à Lyon, mise en place par Christian Schiaretti depuis qu'il dirige le théâtre) ainsi, bien sûr, qu'à la Comédie Française, le statut de cette organisation datant de l'Ancien Régime étant toutefois à part au sein du théâtre public $^{28}$. Enfin, le troisième type de compagnie est celui qu'évoque le terme "collectif», qui fait l'objet, pour sa part, d'une forte valorisation tant par les financeurs que par la critique et par les artistes ${ }^{29}$, en ce qu'il renvoie à une entité marquée par un désir d'horizontalité et de partage de la prise de décision, dans le processus de création (au sein d'un spectacle ou selon les spectacles, tous les collectifs ne créant pas toujours tous leurs spectacles en commun) mais aussi, parfois, dans la gestion des différents aspects du travail et de la vie

27. Pierre-Michel Menger, Portrait de l'artiste en travailleur. Métamorphoses du capitalisme, Paris, Seuil, coll. «La République des idées», 2003.

28. Voir Martial Poirson, «Le Corps de la Troupe: La Comédie-Française, entre gouvernance et autogestion (1680-2016) », dans Bérénice Hamidi-Kim et Séverine Ruset (dir.), Troupes, compagnies, collectifs, ouvr. cité, p. 75-92.

29. Voir Raphaëlle Doyon et Guy Freixe (dir.), Les collectifs dans les arts vivants depuis 1980, ouvr. cité. 
de la compagnie en tant que telle. S'ils s'opposent sur la question du partage de l'auctorialité, ces deux modèles de troupes et de collectifs ont en commun de prétendre lutter contre l'«accélération du temps». Chacune de ces configurations induit évidemment des "chaînes de coopération ${ }^{30}$ » interne différentes et peut favoriser certains types de relations avec les financeurs et d'autres compagnies.

Chacun de ces modèles travaille une forme différente d'auctorialité, et les diverses figures du metteur en scène-directeurtrice de compagnie afférentes à ces modèles font parfois l'objet d'une thématisation dans les œuvres mêmes, témoignant d'un humour qui peut se lire autant comme une réflexivité autocritique que comme une façon d'évacuer la critique extérieure en l'incorporant à son propre discours de justification. Le portrait du metteur en scène en jeune cadre dynamique est ainsi dessiné dans les œuvres de Falk Richter et de Cyril Teste, qui, dans leurs œuvres, investissent les ambivalences propres au «nouveau» capitalisme d'autant plus proliférant et résilient qu'il a su remplacer la menace par la séduction et, de ce fait, d'autant moins étranger aux pratiques de travail existant dans les mondes de l'art et dans le monde du théâtre en particulier ${ }^{31}$. Les spectacles créés par des compagnies qui revendiquent la filiation avec le modèle de la troupe ou, du moins, avec la figure du chef de troupe, même si la pérennité de l'équipe artistique que suppose ce modèle n'est pas toujours concrétisée, jouent pour leur part sur la figure du metteur en scène-dictateur, autoritaire et omnipotent. On pense ici à certains spectacles du Théâtre du Soleil (entre autres, Les naufragés du fol espoir) ou de Vincent Macaigne (Au moinsj'aurai laissé un beau cadavre, en particulier). Quant aux compagnies qui revendiquent ou se voient accoler l'étiquette de "collectif», leurs spectacles mettent volontiers en scène, tout autant que la joyeuse énergie collective, les risques de l'inertie de groupe ou la difficulté à arrêter une décision - on peut penser à Tout ce qui nous reste de la révolution c'est Simon, de l'Avantage du doute ou à certains spectacles du Théâtre du Soleil, comme Et soudain des nuits d'éveil. Il importe en effet de préciser que les compagnies ne ressortissent pas toujours uniquement à un seul

30. Howard S. Becker Chiapello, Les mondes de l'art, Paris, Flammarion, 1988.

31. Voir Bérénice Hamidi-Kim et Armelle Talbot (dir.), "Scène du néomanagement. Avant-propos», thaêtre [En ligne], mis en ligne le 29 janvier 2016, consulté le 31 juillet 2019, URL: https://www.thaetre.com/2016/01/29/scenesdu-neomanagement-avant-propos/. 
de ces modèles. L'un des exemples les plus frappants à cet égard est peut-être justement celui du Théâtre du Soleil, qui relève à première vue du modèle de la troupe, par la captation intégrale de l'auctorialité de l'acte de création par sa directrice artistique (il s'agit de fait de la signature de la compagnie et de la seule figure qui incarne son discours). Pour autant, si sur les plans de la décision et de la vie artistiques il s'agit bel et bien, malgré un processus de création qui assume souvent sa dimension de "création collective», d'une troupe dirigée de main de maitre par Ariane Mnouchkine ${ }^{32}$, la compagnie est structurée depuis sa création en 1959 sur le modèle juridique de la SCOP. Ce modèle prévoit une prise de décision strictement démocratique et horizontale selon le principe: «une personne, une voix», pour les actionnaires de la SCOP qui participent à la prise de décision, et, par ailleurs, cette compagnie pratique l'égalité, ou du moins l'équité des salaires ${ }^{33}$. On peut, dans une moindre mesure, parler d'hybridité dans le cas de Cyril Teste, qui situe sa pratique de metteur en scène au sein d'un collectif artistique, le collectif MxM, «impulsé en 2000 par le metteur en scène Cyril Teste, le créateur lumière Julien Boizard et le compositeur Nihil Bordures», et affirme fonctionner comme un «noyau modulable d'artistes et techniciens, réunis par un même désir de rechercher, créer et transmettre ensemble; de questionner l'individu simultanément en tant que spectateur du réel, de la représentation et de la fiction ${ }^{34}$ », autrement dit, autour d'un projet esthétique commun. Il peut s'agir aussi, dans certains cas, eux aussi moins fréquents, de partager l'outil de production d'une autre façon, non pas au sein du processus de création d'un spectacle, mais selon les spectacles, les différents membres circulant de façon fluide du rôle de metteur en scène à celui d'interprète.

Je voudrais, pour finir, porter plus précisément mon attention sur les collectifs, dont la vitalité tient à la fois au renouveau du socle de légitimité du théâtre public qu'ils permettent, mais aussi au renouvellement des formes scéniques que les artistes, la critique et

32. Pour un témoignage critique savoureux autant qu'affectueux de cette contradiction essentielle du Théâtre du Soleil en général et de la posture d'Ariane Mnouchkine en particulier, on peut se reporter aux Seuls en scène de l'ancien comédien de la troupe Philippe Caubère, notamment Ariane ou l'âge d'or.

33. Voir Ariane Mnouchkine dans Fabienne Pascaud, L'Art du présent, entretiens avec Ariane Mnouchkine, Paris, Plon, 2005, p. 178 et suiv.

34. Voir le site officiel du Collectif MxM, onglet "collectif», consulté le 12 août 2016, URL: http://www.collectifmxm.com/collectif/. 
les pouvoirs publics associent volontiers à ce type d'organisation ${ }^{35}$. Quelques chiffres ${ }^{36}$ confirment les effets incitatifs de cet engouement des pouvoirs publics - et de la critique: $9,9 \%$ des compagnies créées dans les années 1980, 13,4\% des compagnies créées dans les années 1990 et $15,6 \%$ des compagnies créées entre 2000 et 2008 revendiquent une direction artistique collective. De ces chiffres, on peut conclure, d'une part, que l'organisation en collectif est une pratique minoritaire mais non négligeable dans les compagnies et, d'autre part, que ce projet d'une organisation davantage horizontale et partagée du travail artistique a suscité un attrait nouveau dans les années 2000. Comme il a été dit précédemment, ces compagnies sont marquées par un désir d'horizontalité et de fonctionnement polycentré et démocratique, qui se manifeste sur le plan du processus de création et de la signature de la mise en scène ou sur le plan de la vie administrative de la compagnie. Souvent, le désir de travailler ensemble prend sa source dans un sentiment d'appartenance commune né d'un temps de formation partagé, dans une école ou au cours d'un stage, durant lequel se sont créés des valeurs de travail et des sensibilités artistiques communes, mais aussi des liens d'amitié très forts. C'est le cas par exemple pour le D'Ores et déjà, L'Avantage du doute, La Vie Brève, Vous êtes ici ou Les Sans Cou. Le désir d'un travail collectif et égalitaire peut aussi venir du partage de valeurs politiques plus qu'esthétiques, comme pour le collectif intergénérationnel lyonnais les $3 / 8^{37}$.

Né de ce mélange de sentiment d'égalité et de lien affectif très fort, le désir d'horizontalité et de partage de l'auctorialité peut toutefois se manifester à des degrés divers et peut se situer à différents endroits de la chaîne de production interne à la compagnie. Dans beaucoup de cas, l'enjeu premier est le désir d'un processus de création artistique plus collaboratif que celui auquel renvoient les mots

35. Voir aussi à ce sujet Raphaëlle Doyon et Guy Freixe (dir.), Les collectifs dans les arts vivants depuis 1980, ouvr. cité.

36. Voir Daniel Urrutiaguer, Philippe Henry et coll., «Territoires et ressources des compagnies en France», Culture études, $\mathrm{n}^{\circ}$ 1, 2012, p. 1-16; Philippe Henry, «Les compagnies à direction artistique collective en France métropolitaine», art. cité, p. 19 et Un nouveau référentiel pour la culture, Toulouse, Éditions de l'Attribut, 2014, p. 151-152.

37. Voir Bérénice Hamidi-Kim, «Vous avez dit collectif?», dans Raphaëlle Doyon et Guy Freixe (dir.), Les collectifs dans les arts vivants depuis les années 1980, ouvr. cité, p. 51-69. 
«compagnie» ou plus encore «troupe». Il s'agit alors, sinon d'abolir, du moins de flouter et de décentrer la place hégémonique de la figure du metteur en scène individuel. Ce souhait peut se manifester dès le tout début du projet, au moment du choix du sujet de la création: qui est à l'initiative du choix de travailler sur telle ou telle question ou événement, ou autour de tel(s) ou tel(s) texte(s) ou autres œuvres support (roman, poème, scénario de film ou encore peinture), comme cela se fait de plus en plus, revivifiant le goût vitézien de «faire théâtre de tout». L'idée originelle peut être le fait d'un seul, de plusieurs ou de toute l'équipe. Quoi qu'il en soit, tous les artistes qui participeront à la création, et en particulier les artistes interprètes, doivent se l'approprier et mettre au point un "brouillon de rêve ${ }^{38}$ " commun, comme le dit joliment Igor Mendjiski (Les Sans Cou), par des discussions et par la circulation de matériaux favorisant la création d'un imaginaire partagé. La phase suivante est celle où l'ambition d'un travail collectif se manifeste le plus fréquemment et qui déclenche l'appellation ou l'auto-appellation "collectif», quand le spectacle s'élabore à partir d'improvisations collectives des artistes interprètes, cocréateurs et co-auteurs à part entière de l'œuvre. De La Vie Brève au D'Ores et déjà en passant par Les Chiens de Navarre et L'Avantage du Doute ou Les Sans Cou, on ne compte plus le nombre de compagnies qui créent leurs spectacles de la sorte. Il faut noter que l'on peut retrouver ce type de processus de création au sein de la compagnie Louis Brouillard, ce qui fait que le qualificatif de troupe n'est pas parfaitement ajusté à la description du travail de l'équipe de Pommerat ${ }^{39}$. Les situations de jeu et le texte s'élaborent collectivement, à des degrés divers de division du travail auctorial entre interprètes et metteur en scène toutefois. Parfois, le metteur en scène est comme un $D J$ qui «donne des pistes d'improvisation ${ }^{40}$ " puis mixe et sample les improvisations des uns et des autres, ou qui,

38. Igor Mendjiski, «Nous ne sommes pas un collectif, une troupe, plutôt», dans Bérénice Hamidi-Kim et Séverine Ruset (dir.), Troupes, compagnies, collectifs, ouvr. cité, p. 207.

39. D'ailleurs, pour décrire le fonctionnement de la compagnie Louis Brouillard, c'est finalement le terme «collectif» qui a été retenu. Voir Marion Boudier, Anne de Amézaga et Gil Paon, «Un collectif au service d'une vision singulière: la compagnie Louis Brouillard», dans Bérénice Hamidi-Kim et Séverine Ruset (dir.), Troupes, compagnies, collectifs, ouvr. cité, p. 193-204.

40. Frédéric Noaille, entretien réalisé par Bérénice Hamidi-Kim et Séverine Ruset, Villeréal, dans le cadre des représentations de A Kind of Monster, spectacle mis en scène par Jeanne Candel lors du festival Un Festival à Villeréal, 10 juillet 2012. 
comme un couturier, coupe et assemble les pièces. Cela a été le cas par exemple pour Robert Plankett, le spectacle qui a fait découvrir La Vie Brève et plus particulièrement Jeanne Candel. En ce cas, le metteur en scène n'est pas le seul auteur de l'œuvre scénique (ils sont nombreux), mais il est le seul auteur de l'ensemble, le rhapsode de la mise en scène pourrait-on dire. C'est lui qui tranche, comme l'explique Igor Mendjiski:

Au fil du temps, nous avons établi des règles pendant les improvisations: je propose à tel ou tel de jouer dans telle direction. Ensuite, ils improvisent. Puis nous faisons des retours, et c'est sur ce point surtout que les choses ont évolué. Désormais, les comédiens me demandent s'ils peuvent parler de l'improvisation, parce que parfois j'ai besoin de silence pour pouvoir réfléchir et les mots mis immédiatement par d'autres m'empêchent de réfléchir à ma propre réception, et à ma réflexion sur ce qu'il faut garder, retravailler ou enlever. C'est surtout sur le dernier spectacle (Notre crâne comme accessoire) que j'ai eu besoin de poser cette règle, et finalement, de cette façon, les choses sont plus simples pour eux comme pour moi. Parce que ça peut être très difficile la création collective quand ce n'est pas mené par quelqu'un. Les comédiens apprécient cette situation, je pense, parce que par ailleurs ils sont très libres pendant les improvisations et parfois l'ambiance des répétitions ressemble à celle d'une cour de récréation, donc ils comprennent bien que j'ai parfois besoin de silence et de calme justement pour qu'on puisse à la fin aimer tous le spectacle ${ }^{41}$.

Les cas les plus épineux sont ceux où le texte s'écrit dans un va-et-vient entre les improvisations des acteurs sur le plateau et le travail solitaire d'un auteur à sa table, qu'il soit ou non le metteur en scène du projet. L'écriture collective peut alors achopper sur la question symbolique et économique de la signature du texte et sur celle, corollaire, de la répartition des droits d'auteur. Chaque équipe, chaque projet même, compose à sa façon avec cette difficulté. Ainsi, Pommerat signe seul les textes des spectacles, geste vital pour cet artiste d'abord auteur, devenu metteur en scène parce que, s'il avait «attendu qu'on s'intéresse à [s] on écriture, en acceptant ce principe

41. Igor Mendjiski, «Nous ne sommes pas un collectif, une troupe plutôt», art. cité, p. 208. 
de division des tâches, [il] serai[t] devenu fou ou aigri ${ }^{42}$ ». Pour autant, pour certains spectacles, les acteurs touchent également des droits d'auteur ${ }^{43}$, mais ce n'est pas toujours le cas pour l'ensemble des membres de l'équipe artistique. À l'inverse, c'est l'ensemble de la compagnie Les Sans Cou qui a signé l'édition du texte du spectacle Idem. Ce choix ne fait pas non plus consensus dans la mesure où il ne tient pas compte, cette fois, de la part plus grande de travail d'écriture effectuée par le metteur en scène Igor Mendjiski ${ }^{44}$. La situation est encore plus délicate quand un auteur, qui n'est pas le metteur en scène, est associé à l'écriture collective. Ainsi, la dramaturge et autrice Mariette Navarro dit avoir éprouvé des difficultés à faire reconnaître la spécificité de son travail d'écriture auprès du reste de l'équipe d'Elle brûle, spectacle qui a consacré la compagnie Les Hommes approximatifs et plus particulièrement sa metteuse en scène, Caroline Guiela Nguyen ${ }^{45}$. Cet exemple illustre la difficulté structurelle qu'éprouvent les auteurs, pièces rapportées et marginales du procès de production des arts vivants subventionnés français, à voir leur travail reconnu. Néanmoins, la focalisation de la reconnaissance médiatique sur la figure du metteur en scène est ici conforme au désir de la directrice artistique de la compagnie et de son équipe, quelle que soit la dimension collective du geste d'écriture. Il est en revanche d'autres exemples où la compagnie souhaite la reconnaissance de cette dimension collective de la création, mais bute sur les cadres institutionnels. Le collectif de théâtre Lumière d'août insiste ainsi sur la difficulté d'obtenir que les conventions triennales qui les lient avec les collectivités territoriales et avec l'État soient signées par les différents membres du collectif et non par un seul $^{46}$. De même, Samuel Vittoz (directeur de la compagnie Vous êtes ici, puis du Festival de Villeréal) insiste tout à la fois sur la difficulté pour le projet de passer d'une reconnaissance individuelle

42. Joël Pommerat, Théâtres en présence, Arles, Actes Sud-Papiers, coll. «Apprendre», 2007, p. 17.

43. Marion Boudier, Anne de Amézaga et Gil Paon, «Un collectif au service d’une vision singulière», art. cité, p. 200.

44. Igor Mendjiski, «Nous ne sommes pas un collectif, une troupe plutôt», art. cité, p. 209.

45. Entretien personnel avec Mariette Navarro, Lyon, 23 octobre 2011 et entretien public avec Caroline Guiela Nguyen à l'issue de la représentation de Le chagrin, dans le cadre du colloque Troupes, compagnies, collectifs, Centre Dramatique National de Valence, 3 avril 2015.

46. Entretien personnel avec Marine Bachelot-Nguyen, Lyon, 24 octobre 2011. 
à une reconnaissance collective, que ce soit avec les financeurs ou avec la critique $^{47}$, et sur le fait qu'il s'agit d'un impératif politique pour « retrouver les valeurs politiques et démocratiques d'un théâtre dans la cité ${ }^{48}$ » contre le «rapport marchand» qu'incarne à ses yeux la focalisation sur le metteur en scène comme «marque».

Même quand le metteur en scène n'est pas l'auteur principal du texte, cela n'empêche pas forcément la polarisation autour d'une figure centrale, toujours occupée par un metteur en scène, l'exemple archétypal étant celui du D'ores et déjà de Sylvain Creuzevault, qui a d'ailleurs, depuis Le capital et son singe (2015), renoncé à l'appellation "collectif» qui avait accompagné sa fulgurante reconnaissance depuis Notre terreur (2009). Cette figure est à la fois celle qui tranche, durant les répétitions, et celle qui a, au long cours, le statut de directeur artistique de la compagnie, comme l'explique Igor Mendjiski: «Nous nous sommes toujours dit que nous étions une compagnie. Nous ne fonctionnons pas comme un collectif et ce n'est pas un mot que nous revendiquons. Je demande leur avis aux quatre autres membres de la compagnie, nous discutons et réfléchissons beaucoup ensemble, mais je reste le directeur de la troupe ${ }^{49} »$. La différence toutefois avec ce que l'on entend ordinairement par «troupe» est que la figure du metteur en scène est séparée du reste du collectif artistique parce qu'il est le seul à ne pas jouer et peut donc seul occuper la place de «regard extérieur», et non pas par le fait qu'il serait le garant premier de la ligne esthétique de la compagnie et jouerait le rôle de «maître» pour le reste de l'équipe. En ce sens, la compagnie Louis Brouillard est bien davantage une troupe que Les Sans Cou, qui revendique comme figure tutélaire un maitre extérieur à la compagnie, Wajdi Mouawad, ou que La Vie Brève ou la bande du Festival de Villeréal, qui revendiquent pour leur part l'influence fondatrice d'Arpad Schilling.

47. Il explique ainsi avoir été choqué par un article du Monde signé par Brigitte Salino consacrant médiatiquement le Festival de Villeréal par le fait qu'on pouvait y croiser des artistes interprètes liés à quelques grands noms de la nouvelle génération, notamment celui de Sylvain Creuzevault, pourtant absent du festival, mais dont la simple proximité artistique valait un label de qualité (Samuel Vittoz, entretien réalisé par Bérénice Hamidi-Kim et Séverine Ruset, Villeréal, 11 juillet 2012).

48. Samuel Vittoz, entretien réalisé par Bérénice Hamidi-Kim et Séverine Ruset, Villeréal, 11 juillet 2012.

49. Igor Mendjiski, «Nous ne sommes pas un collectif, une troupe plutôt », art. cité, p. 206. 
Ce panorama des différents types de compagnies aura montré combien la question de l'auctorialité du metteur en scène est épaisse, au sens où elle se joue en différents lieux du travail artistique et se manifeste sur différents plans qui ne se recoupent pas: structuration juridique, partage de la prise de décision pour ce qui concerne l'organisation interne ou le processus de création, signature. C'est sur ce dernier point que les résistances à l'égard du partage de l'auctorialité sont les plus fortes, y compris dans les compagnies qui revendiquent le fait de fonctionner «en collectif» et rencontrent pour cette raison même les faveurs de la critique autant que des pouvoirs publics. C'est que, si les valeurs civiques et démocratiques constitutives du socle de justification du théâtre public s'accommodent mal de la figure du directeur d'entreprise, elles font plus qu'accepter la figure du metteur en scène comme créateur d'œuvre et directeur artistique: elles s'y arriment. Je conclurai en suggérant que l'intérêt que portent les compagnies au partage de l'auctorialité de l'acte de création et du discours artistique mais aussi, souvent, politique, est, dans une large mesure, conjoncturel. À une époque de plus en plus marquée par la juridicisation, voire la judiciarisation du débat politique, y compris pour les œuvres d'art, comme en ont témoigné les dernières années les affaires déclenchées par différents spectacles (je pense notamment à Golgotha Picnic de Rodrigo Garcia, Sur le concept du visage du fils de Dieu de Romeo Castellucci, Exhibit B de Brett Bailey, ou Moi la mort je l'aime comme vous aimez la vie de Mohamed Kacimi), la concentration du discours artistique entre les mains d'une seule personne, alors même que l'acte collectif de création a supposé, ou du moins devrait supposer le consentement et l'adhésion de ceux qui y participent, fragilise la légitimité du discours porté par les œuvres. 